segregates from hybrids. Analysis and synthesis involving more than six hundred controlled crosses and selfings correlated with detailed statistical analysis of wild populations have proved the 'herbarium' explanation to be correct.

By linking up taxonomy still more closely with ecology, cytogenetics and other modern developments in biology, it will be improved, its basis and outlook broadened, and more young research workers will be attracted to it. All this is, indeed, happening. The advantages are reciprocal, The cytologist, geneticist and ecologist, both give help to and receive help from the taxonomist. Problems so often need team-work for their solution that it is essential for specialists to know something about the materials, aims and methods of one another's researches. Our divisions are at best artificial conveniences, and taxonomists neither claim absolute national sovereignty over any group or field of research nor recognize such a claim by other biologists.

\section{FRICTION AND LUBRICATION}

\section{ROYAL SOCIETY DISCUSSION}

A

FULL-DAY discussion on friction and lubrication was held in the apartments of the Royal Society at Burlington House on April 19, 1951. The meeting was opened by the president of the Royal Society, Prof. E. D. Adrian, and the leader of the discussion was Dr. F. P. Bowden (Cambridge). Some two hundred people attended, and those presenting papers and participating in the discussion included workers from Australia, France, Holland, Sweden and the United States. The programme fell into three main parts : friction of metals, friction of nonmetals, and boundary and extreme-pressure lubrication.

Metallic Friction. In the opening paper, Dr. Bowden discussed the basic mechanism of friction, and illustrated some of his main points with some simple but striking experiments. He showed that, in general, when surfaces slide on one another, intimate contact occurs between the surfaces over a very small area and in these regions strong junctions are formed - the frictional force being primarily the force required to shear these junctions. The real area of contact, and hence the friction, is proportional to the load and independent of the size of the surfaces. Under suitable conditions these junctions provide strong normal adhesion between the surfaces, and with soft metals such as indium the adhesion is of the same order of magnitude as the frictional force. Lubricant films reduce' the amount of intimate metallic contact and so reduce both the adhesion and friction. Conversely, the removal in a high vacuum of all traces of surface oxide and other contaminant films leads to gross seizure between metals even at room temperature. Most of the frictional energy appears as heat, and because it is localized at the small regions of contact very high local temperatures may be generated at relatively low loads and speeds. This is particularly marked if the surfaces are poor thermal conductors. Finally, the frictional properties of some low.frictional materials were discussed, and it was shown that porous materials impregnated with polytetrafluoroethylene, or molybdenum metal attacked chemically to form molybdenum sulphide in situ on the surface, have very low coefficients of friction up to very high temperatures. It was suggested that a stage is now being reached where it should be possible to construct surfaces having desirable frictional properties for specific practical application.

The five papers which followed dealt primarily with the effect of surface oxide films and surface layers on the frictional behaviour of metals. $R$. Wilson (Cambridge), using electrical resistance measurements between the sliding surfaces, showed that with most metals the natural oxide layer is sufficient to prevent metallic contact at very small loads. The degree of protection depends on a number of factors, such as surface roughness and the thickness of the oxide film, and in particular on the relative hardness of the oxide and metal substrate. Dr. A. J. W. Moore and W. J. McG. Tegart (Commonwealth Scientific and Industrial Research Organization, Australia) described some experiments on a series of copper-beryllium alloys and showed that the friction decreases linearly with increasing hardness. This behaviour was attributed to the more marked plastic deformation of the softer alloys and the increased disruption of the oxide film which therefore ensued. In a second paper these authors showed that, during repeated sliding, oxide is impressed to a considerable depth beneath the surface of the metal. The included oxide particles prevent grain growth in the surface layers during subsequent annealing, and it was suggested that this process may play a part in the formation of the Beilby layer. The importance of the Beilby layer in practical bearing surfaces was stressed by Prof. G. I. Finch and Dr. R. T. Spurr (Imperial College). They showed that in many cases frictional sliding occurs between the oxide rather than the metal, and the fact that the amorphous oxide layer is in general much harder than the underlying metal means that it is better able to prevent intermetallic contact. Dr. R. Courtel (France) described electron diffraction experiments on metal surfaces prepared by grinding in a very high vacuum in the body of the diffraction camera itself. In this way the formation of the oxide film or the adsorption of fatty materials may be directly studied. In the next paper Prof. A. T. Gwathmey, Dr. H. Leidheiser and Dr. G. P. Smith (Virginia, United States) described experiments on the friction and adhesion between large single crystals of copper. When the surface oxides are removed, the coefficient of friction is extremely high $(\mu=25-100)$ and very marked adhesion is also observed.

In the last two papers in this section the mechanism of wear was discussed. Dr. E. M. Trent (Coventry) spoke on the wear of cemented carbide tools. He showed that at high temperatures tungsten carbide forms an alloy with steel so that, in the cutting of steel, tungsten carbide tools wear rapidly. Titanium carbide resists alloy formation and hence greatly reduces the wear. Prof. J. T. Burwell (Massachusetts Institute of Technology) discussed metallic wear under steady-state conditions and showed that the behaviour was consistent with the general picture of formation and shearing of metallic junctions at the regions of real contact. Under his experimental conditions the volume of material worn away was proportional to the load and the length of path traversed. The results suggest that an increase in load produces a proportional increase in the number of welded junctions each of which remains approximately of constant size. Above a certain load, corresponding to a mean pressure about one-third 
the hardness of the pin, a very large increase in the wear is observed, due to the overlapping of the plastic regions around each junction.

In the discussion which followed, Prof. F. E. Simon (Oxford) raised some interesting points on the energetics of the frictional process, and in particular asked for a fundamental mechanism to explain the conversion of friction into heat. Mr. A. W. Crook (Associated Electrical Industries, Ltd., Aldermaston) pointed out that impact experiments on indium indicate that metallic junctions take a finite time to reach their full strength. Mr. P. P. Love (Glacier Metal Co., London) quoted some experimental results on bearing metals impregnated with polytetrafluoroethylene which ran without lubrication under conditions where conventional bearings completely failed.

Friction of Non-metals. In this section some recent experiments on the friction of diamond, graphite and carbon were described in a paper by Dr. F. P. Bowden, J. E. Young and G. W. Rowe (Cambridge). Experiments with surfaces outgassed in vacuo again showed a large increase in friction when the surface films were removed, but the gross seizure observed with metals under comparable conditions did not occur owing to the limited deformation at the regions of contact. Dr. K. V. Shooter (Cambridge) described a study of the frictional properties of plastics. With these materials there is a deviation from Amontons' law at low loads. Apart from this, however, the frictional properties can be explained in terms of adhesion and shearing at the interface exactly as for metals. An important exception is polytetrafluoroethylene, which on account of its molecular structure has an exceptionally low coefficient of friction. In the last paper, Prof. N̦. Gralén (Gothenburg) described some ingenious experiments for determining the friction between single fibres. His investigations show that, as for plastics, the coefficient of friction tends to increase at very small loads. The importance of this work in textile research was emphasized by Mrs. K. R. Makinson (Commonwealth Scientific and Industrial Research Organization, Australia).

Boundary and Lixtreme-Pressure Lubrication. 'This section opened with a survey by Dr. D. Tabor (Cambridge) of the mechanism of boundary lubrication. He showed that, although boundary lubricants enormously reduce the amount of metallic interaction, they do not eliminate it entirely. The most effective lubrication is obtained when the lubricant film is solid and possesses a close-packed structure. When the film melts, a marked increase in friction and metallic interaction occurs. For this reason fatty acids are generally more effective than hydrocarbons or paraffins, since they can react with the metal surface to form metallic soaps of relatively high melting points. These observations have been confirmed in some recent experiments using radioactive metals to detect the amount of metal transferred from one surface to the other as sliding proceeds. A new observation is that, at temperatures above the melting point of the lubricant film, a further deterioration occurs due to the desorption of the lubricant film. At this stage, although the surfaces are flooded with lubricant, the friction and surface damage are comparable with those observed with unlubricated surfaces. These changes, which are reversible on cooling, correspond to changes in state of the lubricant film. Similar changes are observed in electron diffraction studies of the structure and orientation of boundary films.
Dr. J. S. Courtney-Pratt (Cambridge) described an optical method of measuring the thickness of adsorbed layers using the Tolansky multiple-reflexion interferometric technique. The investigation showed directly that molecules of fatty acid, spread by retraction, are adsorbed on the surface as a uniform monomolecular layer. Dr. F. T. Barwell (Mechanical Engineering Research Organization, East Kilbride) discussed the effect of surface structure on the friction of metals sliding under boundary conditions. Surface roughness appears to play a small part; but work-hardening of the surface layers may produce an appreciable increase in the friction. The type of oxide film formed under various conditions may also have a marked effect. Finally, Dr. C. G. Williams (Thornton Research Centre, Chester) spoke on the action of extreme-pressure lubricants. The underlying principle is to use additives which can react with the metal surfaces to form protective films. In general, the additives are inert under less severe conditions of sliding, but at the high temperatures developed at incipient seizure rapid reaction occurs. Metal chloride and metal sulphide films are particularly effective as protective films, and for this reason a large number of extreme-pressure additives contain labile sulphur or chlorine.

In the discussion which followed, Dr. W. Hirst, M. Kerridge and J. K. Lancaster (Associated Electrical Industries, Ltd., Aldermaston) described an investigation of the thickness of the lubricant film necessary for effective boundary lubrication. Using radioactive tracer techniques they have shown that at small loads a single monolayer is sufficient, but at higher loads, where appreciable deformation of the surfaces occurs, considerably thicker films are necessary.

The full discussion will be published in the Proceedings of the Royal Society.

D. TABOR

\section{OBITUARIES}

\section{Dr. J. C. E. Simpson}

James Charles Edward Simpson was educated at St. Edward's School, Oxford, and the University of Liverpool. He graduated in 1929, was awarded the Leverhulme Chemistry Prize and the Campbell Brown Fellowship, and began research under Prof. (now Sir Ian) Heilbron on the chemistry of ergosterol. In 1933 the award of a Commonwealth Fund Fellowship took him to the Rockefeller Institute, New York, where he studied steroid sapogenins with Dr. W. A. Jacobs. He returned to England in 1935 and became an assistant lecturer at King's College, London, where for four years his work was mainly in the triterpene field, with particular reference to $\beta$-boswellic acid and $\beta$-amyrin.

From 1939 Dr. Simpson was a temporary lecturer in the Durham Division of the University of Durham. There he became interested in the chemistry of cinnoline, and this system was studied together with related antimalarial agents. In 1945 , he was awarded the D.Sc. degree of the University of Liverpool and at the beginning of that year went as I.C.I. Fellow to the Chemotherapy Department of the Liverpool School of Tropical Medicine. Collaboration with the director, Dr. E. M. Lourie, on the chemotherapy of trypanosomiasis was continued after 1946, when Dr. Simpson was appointed to the scientific staff of the Medical Research Council. A comparative study of 